\title{
Long-term Disease-free Survival after Trimodality Treatment of Recurrent Hepatocellular Carcinoma Involving the Inferior Vena Cava and Right Atrium
}

\author{
Sunmin Park', Won Sup Yoon', Hyung Joon Yim², Chai Hong Rim ${ }^{1}$ \\ 'Department of Radiation Oncology, ${ }^{2}$ Division of Gastroenterology and Hepatology, Department of Internal Medicine, Korea University \\ Ansan Hospital, Ansan, Korea
}

Received Mar. 22, 2019

Revised Mar. 22, 2019

Accepted May 14, 2019
Hepatocellular carcinoma (HCC) involving the inferior vena cava (IVC) and/or right atrium (RA) is a rare and intractable disease. A standard treatment has not been established yet, owing to the rarity of disease and difficulties in the therapeutic treatment. Herein, we report the case of a patient who had recurrent HCC (after a prior lobectomy) involving both IVC and RA and underwent multimodality treatments including external beam radiotherapy and transarterial chemotherapy, followed by sorafenib treatment. The disease was well controlled with local treatments and sustained for 7 years until last follow-up after the systemic treatments. Our case shows a possibility of long-term survival for patients affected by HCC involving IVC and/ or RA, after a rigorous multimodality treatment strategy. (J Liver Cancer 2019;19:149-153)

Keywords: Liver neoplasm; Inferior vena cava; Right atrium; Radiotherapy

\section{INTRODUCTION}

Hepatocellular carcinoma (HCC) involving the inferior vena cava (IVC) or the right atrium (RA) is rare, occurring in less than $4 \%$ of all HCC patients. ${ }^{1}$ The prognosis is poor with an expected survival time less than 3 months, if left untreated. ${ }^{2}$ A standard treatment has not been established yet, as the incidence of the disease is rare and the treatment has a negative impact on the pulmonary and systemic circulation.

Although the Barcelona Clinic Liver Cancer staging criteria recommend sorafenib for treating HCC involving major vessels, ${ }^{3}$ local treatments have been commonly performed in-

\footnotetext{
Corresponding author : Chai Hong Rim

Department of Radiation Oncology, Korea University Ansan Hospital, 123 Jeokgeum-ro, Danwon-gu, Ansan 15355, Korea

Tel. +82-31-412-6850, Fax. +82-31-412-6851

E-mail; crusion3@naver.com

https://orcid.org/0000-0001-7431-4588
}

stead, compensating for the poor tumor response with an expected longer survival. ${ }^{4}$ External beam radiation therapy (EBRT) is a local treatment that is less affected by the tumor location unlike other local treatments. ${ }^{5}$ In particular, radiofrequency ablation (RFA) or surgery is difficult for tumors involving major vessels or for those present in their vicinity, whereas EBRT is relatively more feasible. The estimated tolerance dose of radiation for major vessels, commonly extrapolated from studies on central lung tumors, ${ }^{6}$ exceeds $100 \mathrm{~Gy}$, which is enough for efficient control of the tumor. ${ }^{7}$

Pioneering researchers tried using local therapies such as transarterial chemoembolization (TACE) and EBRT achieving successful tumor response rates for HCCs involving IVC and/or RA. ${ }^{8,9}$ Nonetheless, the life expectancy of patients with HCCs involving IVC and/or RA is still approximately 1 year, with most patients dying within 2 years even after rigorous local treatments. ${ }^{10}$ We shall review and report here a case of HCC involving IVC and RA treated with active local 
treatments, including EBRT, in which the patient survived for up to 7 years in a disease-free state until the last follow-up.

\section{CASE REPORT}

A 62-year-old man experienced upper-right abdominal pain after a fall and was referred to us by a private clinic after a fracture of the 10th rib was suspected following an X-ray examination. We performed abdominal ultrasonography and incidentally found a liver mass. The patient reported no history of viral hepatitis or undergoing screenings. Until he was admitted, he consumed two bottles of Soju (Korean distilled liquor with an alcohol content of 15-20\%) 3-4 days a week and smoked a pack of cigarettes every day for 42 years. He was on medications for treating hypertension. Blood analyses revealed the following parameters: white blood cell (WBC), 6,210/ $\mathrm{mm}^{3}$; hemoglobin (Hgb), $11.6 \mathrm{~g} / \mathrm{dL}$; and platelet,
$225,000 / \mathrm{mm}^{3}$. Biochemical analyses showed the following: total protein, $7.7 \mathrm{~g} / \mathrm{dL}$; albumin, $4.1 \mathrm{~g} / \mathrm{dL}$; blood urea nitrogen (BUN), $13 \mathrm{mg} / \mathrm{dL}$; creatinine $(\mathrm{Cr}), 1.0 \mathrm{mg} / \mathrm{dL}$; aspartate aminotransferase/alanine aminotransferase (AST/ALT), 202/45 IU/L; alkaline phosphatase (ALP), 300 IU/L; total bilirubin, $0.5 \mathrm{mg} / \mathrm{dL}$ (direct bilirubin $0.3 \mathrm{mg} / \mathrm{dL}$ ); and prothrombin time/international normalized ratio (PT/INR): 0.84. Viral hepatitis serologic tests showed positivity for HBsAg, anti-HBs Ab, IgG anti-HBc, and negativity for the antihepatitis $\mathrm{C}$ virus antibody ( $\mathrm{HCV} \mathrm{Ab}$ ), while hepatitis $\mathrm{B}$ virus (HBV) deoxyribonucleic acid (DNA) was $355 \mathrm{IU} / \mathrm{mL}$. Among tumor markers, alpha-fetoprotein (AFP) was 11.2 $\mathrm{ng} / \mathrm{mL}$, while protein induced by vitamin $\mathrm{K}$ absence or antagonist-II (PIVKA-II) was $>2,000 \mathrm{mAU} / \mathrm{mL}$. Primovist magnetic resonance imaging (MRI) showed a $12 \mathrm{~cm}$ mass in the right liver lobe with an arterial enhancement and delayed washout, as shown in Fig. 1A. The patient had a Child-Pugh
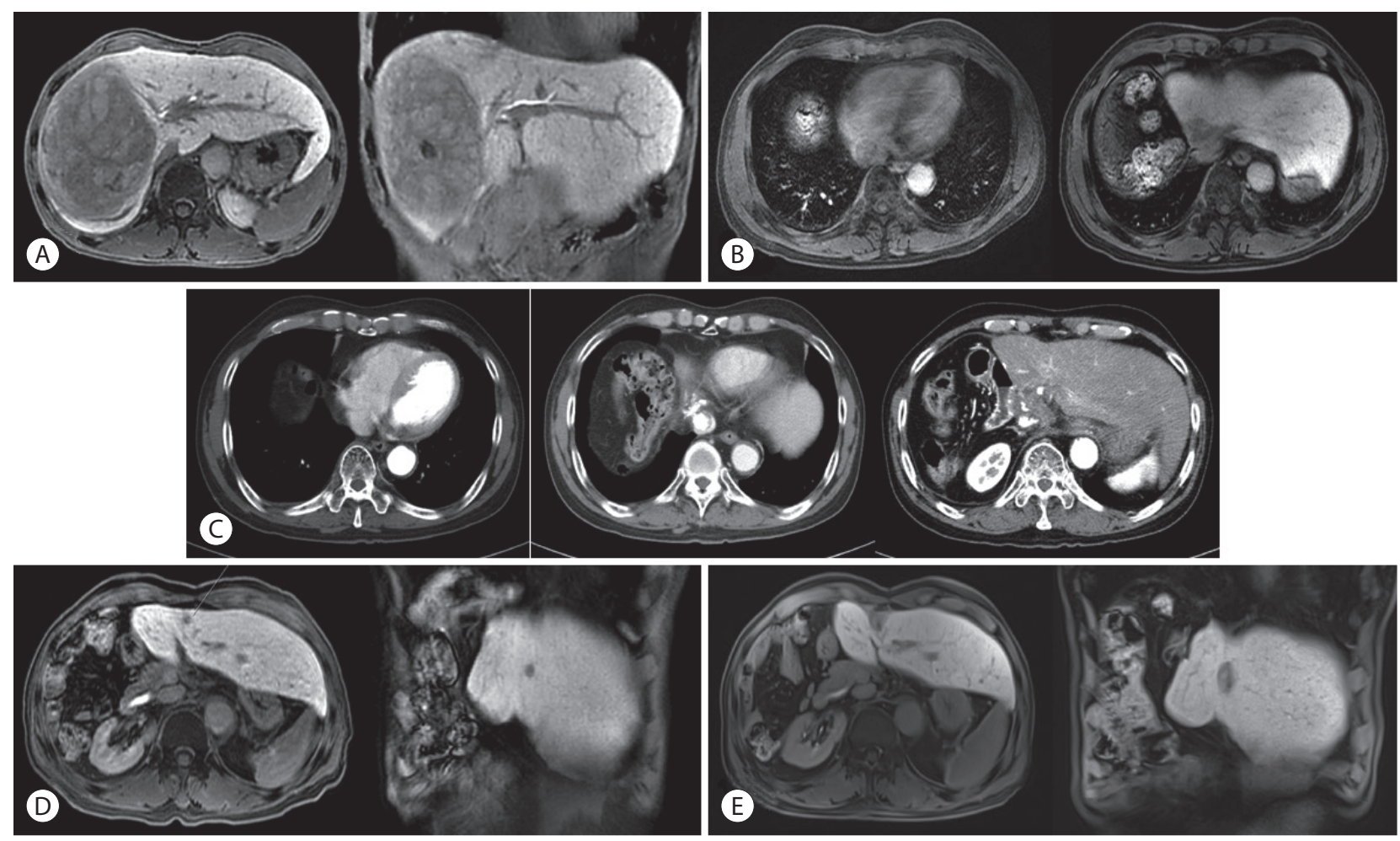

Figure 1. Imaging studies at the initial diagnosis and at different follow-ups. (A) Liver magnetic resonance imaging at the initial diagnosis. A mass $12 \mathrm{~cm}$ in size was noted in the right lobe of the liver. The tumor showed arterial enhancement and a delayed washout pattern. (B) Two years after the surgery, HCC recurred in segment 4 with tumor thrombus in the inferior vena cava (IVC) and right atrium (RA). (C) Three months after external beam radiotherapy (EBRT) and 3 cycles of transarterial chemotherapy (TACE), the thrombus in the RA disappeared, the IVC was well-lipiodolized, but a filling defect was seen around the IVC. Sorafenib was administered after EBRT and TACE. (D) Three years and 4 months post-TACE and EBRT followed by sorafenib, recurrence at segment 3 was observed. (E) Radiofrequency ablation (RFA) was performed and a good ablation was seen in the follow-up image 3 months after the RFA. 
score of five, for which he was classified as class A. His Eastern Cooperative Oncology Group (ECOG) performance status score was 0. The tumor was graded as a Barcelona Clinic Liver Cancer (BCLC) stage B, for which he underwent right hepatectomy. After the surgery, the tumor size was $14.0 \times 9.0$ $\mathrm{cm}$ and did not involve the liver capsule, but we observed the presence of vascular tumor emboli and a resection margin of less than $0.1 \mathrm{~cm}$. Afterwards, the patients had regular clinical and radiologic follow-ups at 3- or 6-month intervals, respectively, for which he underwent liver dynamic computed tomography or MRI and PIVKA-II without further treatment. Imaging showed no evidence of recurrence, but PIVKA-II levels increased continuously after one year from the surgery. Two years after the surgery, HCC recurred in segment 4 with tumor thrombus in the IVC and in the RA, with PIVKA-II levels of 1,463 mAU/mL, as shown in Fig. 1B. Blood analyses revealed the following parameters: WBC, 4,100/ $\mathrm{mm}^{3} ; \mathrm{Hgb}$, $12.8 \mathrm{~g} / \mathrm{dL}$; and platelets, $138,000 / \mathrm{mm}^{3}$. Biochemical analyses showed the following: total protein, $6.7 \mathrm{~g} / \mathrm{dL}$; albumin, $4.0 \mathrm{~g} / \mathrm{dL}$; BUN, $13.6 \mathrm{mg} / \mathrm{dL}$; Cr, $0.99 \mathrm{mg} / \mathrm{dL}$; AST/ALT, 24/15 IU/L; ALP, $48 \mathrm{IU} / \mathrm{L}$; total bilirubin, $0.42 \mathrm{mg} / \mathrm{dL}$ (direct bilirubin $0.10 \mathrm{mg} / \mathrm{dL}$ ); and PT/INR, 1.03. Viral hepatitis serologic tests showed positivity for anti-HBs Ab and IgG anti-HBc, negativity for HBsAg and anti-HCV Ab, and HBV DNA levels $<20 \mathrm{IU} / \mathrm{mL}$. Among tumor markers, AFP was $2.3 \mathrm{ng} / \mathrm{mL}$. At the time of recurrence, the patient had a Child-Pugh score of five, for which he was classified as class A again. His ECO performance status score was 0 . The tumor was graded as BCLC stage C (advanced), due to the IVC invasion. In echocardiography, the ejection fraction was 69\% without any

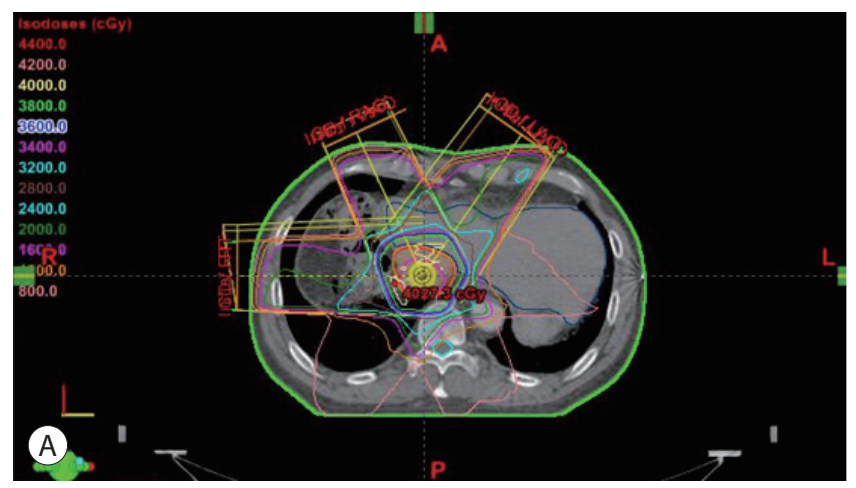

symptom, but a tumor thrombus was involved from the IVC to the RA junctions. Ascites were not evident, and the liver surface was smooth.

We planned a combination therapy of TACE and EBRT. We performed TACE every 4 weeks with the following protocol: first, we infused $2 \mathrm{mg} / \mathrm{kg}$ cisplatin (Dong-A Pharmaceutical, Seoul, Korea) as chemotherapeutic agent. Second, we embolized feeder arteries until we achieved arterial flow stasis with an emulsion of 5 to $10 \mathrm{~mL}$ of cisplatin and iodized oil (Lipiodol Ultra-Fluide; Laboratoire Guerbet, Aulnay sous Bois, France). Lastly, we applied an absorbable gelatin sponge (Gelfoam; Upjohn, Kalamazoo, MI, USA). The feeder arteries were embolized until arterial flow stasis was achieved. For EBRT planning, we calculated the gross tumor volume using a CT scan and a 20-minute delayed MRI, which were fused with a rigid registration method. We applied an abdominal compressor to the umbilical area to diminish respiratory movement, and we designed the internal target volume (ITV) respecting the respiratory movement, tracked by a Real-Time Position Management system (Varian Medical System, Palo Alto, CA, USA). Then, we expanded the ITV by 5-7 mm to obtain the planning target volume (PTV). We administered a radiation dose of $40 \mathrm{~Gy}$ in 16 fractions to the PTV. Following the Korean Liver Cancer Study Group guidelines, we kept the mean normal liver dose below $28 \mathrm{~Gy}$ (an equivalent doses of 2 Gy per fraction), and more than $700 \mathrm{~mL}$ of normal liver volume received a dose below $15 \mathrm{~Gy}$. This dose scheme was delivered to PTV every day. The duodenum $\mathrm{D}_{1 \mathrm{cc}}$ (the largest dose received by at least $1 \mathrm{~mL}$ of duodenum) was $23.5 \mathrm{~Gy}$, while the mean PTV dose was $40.1 \mathrm{~Gy}$.

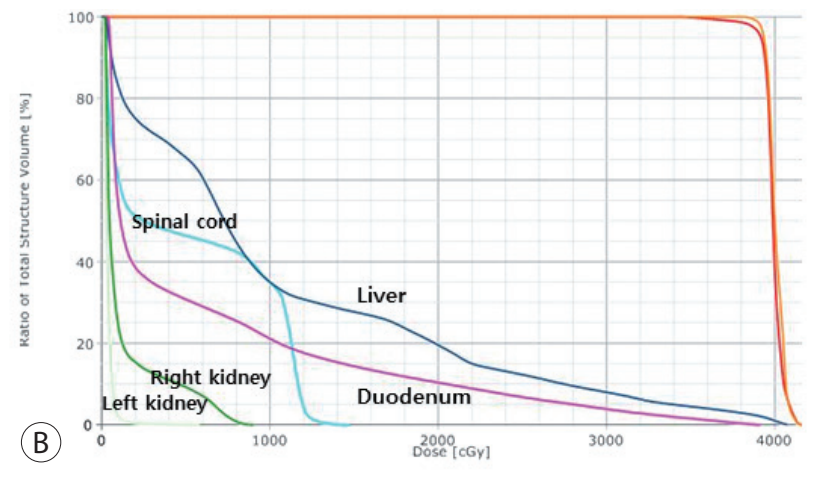

Figure 2. External beam radiotherapy planning to treat the tumor at segment 4 with a right atrium and inferior vena cava thrombus. (A) Scheme of the radiation dose distribution. (B) Dose-volume histogram showing the doses received by organs and the relevant volumes. 
We performed the planning using the Eclipse 8.9 (Varian Medical System) software. The plan and the dose-volume histogram is shown in Fig. 2. Three months after EBRT and 3 cycles of TACE, as shown in Fig. 1C, all thrombi in RA were eliminated, the suprahepatic IVC thrombus was welllipiodolized with no arterial enhancement although a filling defect was present in IVC. AFP levels significantly dropped from $11.2 \mathrm{ng} / \mathrm{mL}$ to a normal level of $2.3 \mathrm{ng} / \mathrm{mL}$. PIVKA-II levels also normalized at $37 \mathrm{mAU} / \mathrm{mL}$. By definition of the modified Response Evaluation Criteria in Solid Tumors, this was considered a complete response. After TACE and EBRT treatment, we administered sorafenib (200 mg, b.i.d). Although during sorafenib maintenance we did not observe any recurrence on the follow-up images, we had to stop the medication because the patients reached a grade 3 of gastrointestinal toxicity (diarrhea and vomiting) in the Common Terminology Criteria for Adverse Events (CTCAE) 4.03 scale, 2 years and 6 months after the first administration of sorafenib. After three years and four months since the discontinuation of sorafenib, we saw a recurrence in segment 4 in a follow-up MRI, as shown in Fig. 1D. Thus, we performed the RFA procedure. Afterwards, the patient underwent regular radiological imaging and tumor-marker followup without further treatments and continued to show a no evidence of disease status without recurrence 7 years after the TACE and EBRT treatment, about 10 years after the first diagnosis as shown in Fig. 1E.

\section{DISCUSSION}

Recently, Samsung Medical Center, one of the high-volume centers in Korea, published a study showing that HCC patients who were treated with a multidisciplinary approach had a higher survival rate than the control group. ${ }^{11}$ As there are different staging systems for grading HCC, multiple treatment options are available for even for the same stage of disease. ${ }^{12}$ Some researchers describe those considerations as a 'fierce battle'; ${ }^{13}$ however, we show here that a combined therapy strategy can cure HCC previously considered intractable.

Spatial cooperation between TACE and EBRT could maximize local control, as TACE could eradicate lesions too small to be easily observed, EBRT removes the main tumor mass. Furthermore, the anticancer drugs used for TACE have a radiosensitizing effect. ${ }^{14}$ Treatment with sorafenib played an important role in controlling possible subclinical disease and residual tumor under control, due the inhibition of growth factors and Raf. ${ }^{15}$ Unlike other major cancers, for HCC the role of systemic treatment or radiotherapy has not been established for HCC until recently. Therefore, it is necessary to establish an optimal combination strategy of therapy modalities to maximize the treatment efficacy.

In two relatively large trials including patients affected by HCC with major vessel invasion, patients with HCC involving IVC and/or RA had a superior overall survival compared to those with HCC involving portal vein. ${ }^{8,16}$ In a recent pooled meta-analysis, the tumor response rate to EBRT of HCC involving IVC and/or RA exceeded 60\%, and the local control rate was as high as $84 \% .{ }^{10} \mathrm{~A}$ theoretical concern has been raised, that treating HCC involving IVC and/or RA with potent local treatments such as EBRT could lead to pulmonary embolism or sudden death due to a dropped-out necrotized stalk. ${ }^{17}$ However, only one case of pulmonary embolism was reported in a meta-analysis of 192 patients, and the rate of grade $\geq 3$ complications was only $1.2 \% .{ }^{10}$ Hence, we consider EBRT for HCC involving IVC and/or RA a feasible local treatment strategy.

In a study by Hou et al. ${ }^{16}$, intrahepatic tumor numbers and their control status affected the survival rate of patients with HCC involving IVC. Koo et al. ${ }^{17}$ reported that liver function and the invasion of the portal vein are significant factors affecting the survival. Two studies indicated IVC thrombosis response and combined treatment of TACE and EBRT as strong predictors of survival. ${ }^{16,17}$ Although a definitive cure might be difficult even for cases satisfying all the above prognosticators, our case suggests the possibility that active multimodality treatment for localized HCC involving IVC and/ or RA could result in long-term survival.

Although we did not need any systemic agents other than sorafenib for this case, regorafenib ${ }^{18}$ and carbozantinib ${ }^{19}$ have successfully shown survival benefit and can be used as second-line drugs if sorafenib fails. Many ongoing trials are evaluating the efficacy and feasibility of combined treatments 
including locoregional and novel systemic modalities. ${ }^{20}$ Our reported case could contribute to the establishment of updated optimization of multimodality therapeutic strategies including locoregional and systemic treatments, to improve the chances of curing intractable HCC cases.

\section{SUPPLEMENTARY MATERIAL}

Supplementary datas can be found with this article online

http://www.e-jlc.org/html/https://doi.org/10.17998/ jlc.19.2.149.

\section{Conflicts of Interest}

The authors have no conflicts to disclose.

\section{REFERENCES}

1. Lee IJ, Chung JW, Kim HC, Yin YH, So YH, Jeon UB, et al. Extrahepatic collateral artery supply to the tumor thrombi of hepatocellular carcinoma invading inferior vena cava: the prevalence and determinant factors. J Vasc Interv Radiol 2009;20:22-29.

2. Llovet JM, Bustamante J, Castells A, Vilana R, Ayuso M, Sala M, et al. Natural history of untreated nonsurgical hepatocellular carcinoma: rationale for the design and evaluation of therapeutic trials. Hepatology 1999;29:62-67.

3. European Association For The Study Of The Liver, European Organisation For Research And Treatment Of Cancer. EASL-EORTC clinical practice guidelines: management of hepatocellular carcinoma. J Hepatol 2012;56:908-943.

4. Rim CH, Seong J. Application of radiotherapy for hepatocellular carcinoma in current clinical practice guidelines. Radiat Oncol J 2016;34:160-167.

5. National Comprehensive Cancer Network. NCCN clinical practice guidelines in oncology, hepatobiliary cancers 2017. Fort Washington: National Comprehensive Cancer Network, 2017.

6. National Comprehensive Cancer Network. NCCN clinical practice guidelines in oncology, non-small cell lung cancers 2017. Fort Washington: National Comprehensive Cancer Network, 2017.

7. $\operatorname{Rim} \mathrm{CH}, \mathrm{Kim}$ CY, Yang DS, Yoon WS. Comparison of radiation therapy modalities for hepatocellular carcinoma with portal vein thrombosis: a meta-analysis and systematic review. Radiother Oncol 2018;129:112-122.

8. Zeng ZC, Fan J, Tang ZY, Zhou J, Qin LX, Wang JH, et al. A comparison of treatment combinations with and without radiotherapy for hepatocellular carcinoma with portal vein and/or inferior vena cava tumor thrombus. Int J Radiat Oncol Biol Phys 2005;61:432443.

9. Yoon SM, Lim YS, Won HJ, Kim JH, Kim KM, Lee HC, et al. Radiotherapy plus transarterial chemoembolization for hepatocellular carcinoma invading the portal vein: long-term patient outcomes. Int J Radiat Oncol Biol Phys 2012;82:2004-2011.

10. Rim CH, Kim CY, Yang DS, Yoon WS. External beam radiation therapy to hepatocellular carcinoma involving inferior vena cava and/ or right atrium: a meta-analysis and systemic review. Radiother Oncol 2018;129:123-129.

11. Sinn DH, Choi GS, Park HC, Kim JM, Kim H, Song KD, et al. Multidisciplinary approach is associated with improved survival of hepatocellular carcinoma patients. PLoS One 2019;14:e0210730.

12. Korean Liver Cancer Study G. National Cancer Center K. 2014 Korean Liver Cancer Study Group-National Cancer Center Korea practice guideline for the management of hepatocellular carcinoma. Korean J Radiol 2015;16:465-522.

13. Sanduzzi-Zamparelli M, Bruix J. Locoregional versus systemic therapy-robust positive data remain elusive. Nat Rev Clin Oncol 2018;15:537.

14. Seong J, Kim SH, Suh CO. Enhancement of tumor radioresponse by combined chemotherapy in murine hepatocarcinoma. J Gastroenterol Hepatol 2001;16:883-889.

15. Llovet JM, Ricci S, Mazzaferro V, Hilgard P, Gane E, Blanc JF, et al. Sorafenib in advanced hepatocellular carcinoma. N Engl J Med 2008;359:378-390.

16. Hou JZ, Zeng ZC, Zhang JY, Fan J, Zhou J, Zeng MS. Influence of tumor thrombus location on the outcome of external-beam radiation therapy in advanced hepatocellular carcinoma with macrovascular invasion. Int J Radiat Oncol Biol Phys 2012;84:362-368.

17. Koo JE, Kim JH, Lim YS, Park SJ, Won HJ, Sung KB, et al. Combination of transarterial chemoembolization and three-dimensional conformal radiotherapy for hepatocellular carcinoma with inferior vena cava tumor thrombus. Int J Radiat Oncol Biol Phys 2010;78:180-187.

18. Bruix J, Qin S, Merle P, Granito A, Huang YH, Bodoky G, et al. Regorafenib for patients with hepatocellular carcinoma who progressed on sorafenib treatment (RESORCE): a randomised, doubleblind, placebo-controlled, phase 3 trial. Lancet 2017;389:56-66.

19. Abou-Alfa GK, Meyer T, Cheng AL, El-Khoueiry AB, Rimassa L, Ryoo BY, et al. Cabozantinib (C) versus placebo (P) in patients (pts) with advanced hepatocellular carcinoma (HCC) who have received prior sorafenib: results from the randomized phase III CELESTIAL trial. J Clin Oncol 2018;36(4-suppl):207.

20. $\operatorname{Rim} \mathrm{CH}$, Yoon WS. Leaflet manual of external beam radiation therapy for hepatocellular carcinoma: a review of the indications, evidences, and clinical trials. Onco Targets Ther 2018;11:2865-2874. 


\section{Journal of Liver Cancer}

Volume 19 Number 2, September 2019

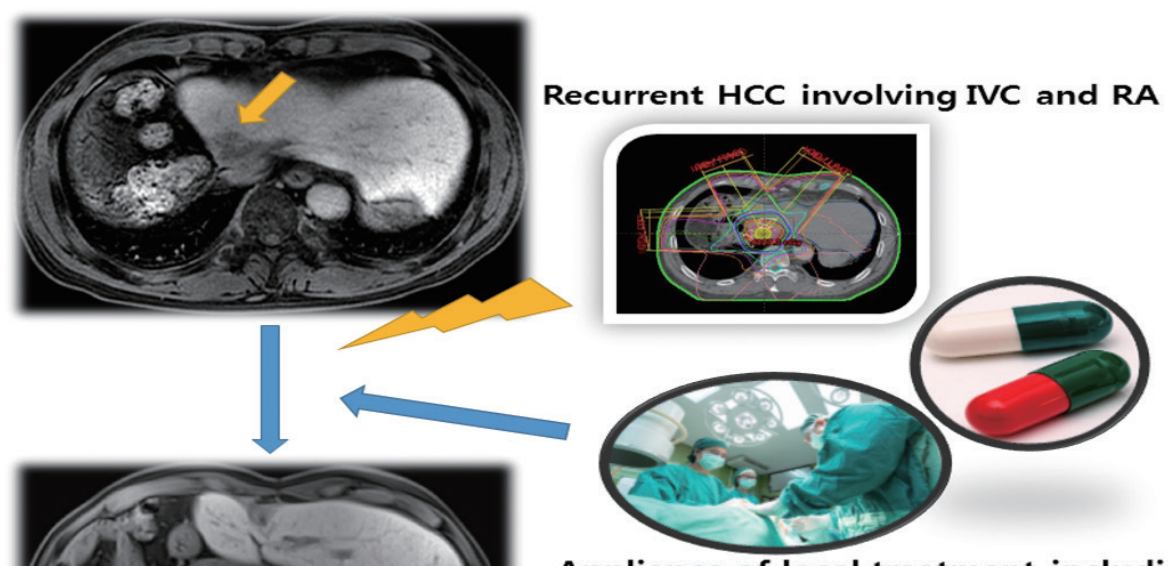

Appliance of local treatment including EBRT, TACE Followed by sorafenib

Alive with NED status for seven years

Supplementary Figure 1. HCC, hepatocellular carcinoma; IVC, the inferior vena cava; RA, right atrium; EBRT, external beam radiation therapy; TACE, transarterial chemoembolization; NED, no evidence of disease. 